\title{
Grouping and characterization of putative glycosyltransferase genes from Panax ginseng Meyer
}

\author{
Altanzul Khorolragchaa, Yu-Jin Kim, Shadi Rahimi, Johan Sukweenadhi, Moon-Gi Jang, Deok-Chun Yang * \\ Department of Oriental Medicinal Materials and Processing, College of Life Science, Kyung Hee University, Seocheon, Giheung-gu, Yongin-si, Gyeonggi-do 446-701, South Korea
}

\section{A R T I C L E I N F O}

Article history:

Accepted 18 July 2013

Available online 24 August 2013

\section{Keywords:}

UDP-glycosyltransferase

Panax ginseng

EST

Methyl jasmonate

\begin{abstract}
A B S T R A C T
Glycosyltransferases are members of the multigene family of plants that can transfer single or multiple activated sugars to a range of plant molecules, resulting in the glycosylation of plant compounds. Although the activities of many glycosyltransferases and their products have been recognized for a long time, only in recent years were some glycosyltransferase genes identified and few have been functionally characterized in detail. Korean ginseng (Panax ginseng Meyer), belonging to Araliaceae, has been well known as a popular mysterious medicinal herb in East Asia for over 2000 years. A total of 704 glycosyltransferase unique sequences have been found from a ginseng expressed sequence tag (EST) library, and these sequences encode enzymes responsible for the secondary metabolite biosynthesis. Finally, twelve UDP glycosyltransferases (UGTs) were selected as the candidates most likely to be involved in triterpenoid synthesis. In this study, we classified the candidate P. ginseng UGTs (PgUGTs) into proper families and groups, which resulted in eight UGT families and six UGT groups. We also investigated those gene candidates encoding for glycosyltransferases by analysis of gene expression in methyl jasmonate (MeJA)-treated ginseng adventitious roots and different tissues from four-year-old ginseng using quantitative reverse transcriptase-polymerase chain reaction (RT-PCR). For organ-specific expression, most of PgUGT transcription levels were higher in leaves and roots compared with flower buds and stems. The transcription of PgUGTs in adventitious roots treated with MeJA increased as compared with the control. PgUGT1 and PgUGT2, which belong to the UGT71 family genes expressed in MeJA-treated adventitious roots, were especially sensitive, showing 33.32 and 38.88-fold expression increases upon $24 \mathrm{~h}$ post-treatments, respectively.
\end{abstract}

(C) 2013 Elsevier B.V. All rights reserved.

\section{Introduction}

Korean ginseng (Panax ginseng Meyer) is a typical medicinal plant and has been used as a traditional medicine since the ancient times. The major active component of ginseng roots is ginsenoside, a triterpenoid saponin. Saponins have been shown to have many beneficial bioactive effects on human health, such as anti-tumor, anti-stress, anti-aging, and improving immune function (Cheng et al., 2005; Lee et al., 2009; Park and Cho, 2009; Yuan et al., 2012). Due to their broad pharmaceutical properties, special attention has been paid to genes involved in ginsenoside biosynthesis for large-scale production. Ginsenosides are synthesized by the cyclization of 2,3-oxidosqualene via the mevalonate pathway. This cyclization occurs at the branching point leading to oleanane- and dammarene-type ginsenosides. Dammarene-type triterpenes undergo further modifications such as oxidation/hydroxylation, substitution, and glycosylation leading

Abbreviations: EST, expressed sequence tags; GT, glycosyltransferase; MeJA, methyl jasmonates; UGT, UDP glycosyltransferases; RT-PCR, reverse transcriptase-polymerase chain reaction.

* Corresponding authors at: Korean Ginseng Center and Ginseng Genetic Resource Bank, Kyung Hee University, 1 Seocheon, Giheung-gu Yongin-si, Gyeonggi-do 449-701, South Korea. Tel.: +82312012100; fax: +82312022687.

E-mail address: dcyang@khu.ac.kr (D.-C. Yang). to protopanaxadiol- and protopanaxatriol-type ginsenosides. Despite commercial interest in ginsenosides, very little is known about the genes involved in the biosynthetic pathway of triterpenoid saponins (Woo et al., 2004). Cytochrome P450 plays a hydroxylation role resulting in protopanaxadiol and protopanaxatriol type ginsenosides (Jung et al., 2003).

Glycosylation is the last step in the biosynthesis of the secondary metabolite. The glycosylation process is catalyzed by glycosyltransferase (GTs), which are highly divergent and polyphyletic, and belong to a multigene family in plant organisms. GTs from diverse species have been classified into 94 families based on amino acid sequence similarities, catalytic mechanisms, and the presence of a conserved sequence motif (http://www.cazy.org/GlycosylTransferases.html). Among them, the GT family 1 is the largest, often referred to as UDPglycosyltransferases (UGTs), and catalyzes the transfer of a glycosyl moiety from UDP sugars to a diverse array of substrates, including hormones, secondary metabolites, and xenobiotics such as pesticides and herbicides (Barvkar et al., 2012; Ross et al., 2001). UGTs play an important role in the stabilization, enhancement of water solubility, and deactivation/detoxification of natural products, leading to regulation of metabolic homeostasis, detoxification of xenobiotic, and the biosynthesis, storage, and transport properties of secondary metabolites. UGTs contain a conserved protein domain (PROSITE accession number 